MATEC Web of Conferences 24,04006 (2015)

DOI: $10.1051 /$ matec conf/ 20152404006

(C) Owned by the authors, published by EDP Sciences, 2015

\title{
Wind-induced vibration experiment on solar wing
}

\author{
Yukio Tamura1 ${ }^{1}$ Yong Chul Kim²,a, Akihito Yoshida² and Takashi Itoh ${ }^{3}$ \\ ${ }^{1}$ School of Civil Engineering, Beijing jiaotong University, 3 Shangyuancun Xizhimenwai, Beijing, China \\ ${ }^{2}$ Wind Engineering Research Center, Tokyo Polytechnic University, 1583 liyama Atsugi, Kanagawa, Japan \\ ${ }^{3}$ Photovoltaic Power Section, JFE Technos Co.Ltd., 2-1 Suehiro-cho Tsurumi-ku Yokohama, Kanagawa, Japan
}

\begin{abstract}
This paper describes wind tunnel experimental results of wind-induced responses of a solar wing system, and investigates its aeroelastic instability using a scaled model. The model comprised 12 solar wing units, each supported by 2 cables. The gaps between units were set constant. Two sag ratios (i.e. sag/span length) were adopted. The wind speed was varied from 0 to $16 \mathrm{~m} / \mathrm{s}$, and 18 different wind speeds were used. From the experiment, when the sag was $2 \%$, a sudden increase in fluctuating displacement was found near a mean wind speed of $10 \mathrm{~m} / \mathrm{s}$ at a wind direction of $40^{\circ}$. A sudden increase in fluctuating displacements was also found near a mean wind speed of $1 \mathrm{~m} / \mathrm{s}$ when the wind direction was larger than $60^{\circ}$. When the sag increased to $5 \%$, some differences among units in mean displacements were found and complicate vibration in fluctuating displacement at low wind speed was observed.
\end{abstract}

\section{Introduction}

Construction of $1000 \mathrm{~kW}$ solar power generating systems is increasing as clean alternative energy sources. Because there is no worry about solar power becoming depleted, and that it does not emit carbon dioxide, it has been attracting increasing attention. Solar cell shipments in Japan have also increased rapidly, almost 14 times in the past 10 years. Most solar power generating systems in Japan are ground mounted as solar farms (i.e. those generating more than $1000 \mathrm{~kW}$ ). However, these systems require huge areas of land, which are difficult to find in Japan because it is so mountainous. In some mountainous countries, new generating systems called solar wings have begun to be constructed $[1,2]$. In a solar wing system, solar wing units are suspended in the air by 2 cables. However, there has been no systematic research on wind-induced responses of solar wing systems. Thus, the aerodynamic stability of these structures needs to be checked in a wind tunnel.

This paper describes wind tunnel experimental results of wind-induced responses of a solar wing system, and investigates its aeroelastic instability using a 1/13.3 scaled model. The model comprised 12 solar wing units, each supported by 2 cables. Each unit was $74.7 \mathrm{~mm}$ wide, $25.5 \mathrm{~mm}$ high and $755.5 \mathrm{~mm}$ long in model scale. Two sag ratios (i.e., sag/span length) were adopted. Three laser displacement transducers were installed under the turntable to measure the vertical displacements. Strain gauges were also installed on two of the model's columns to measure tension in the cables. The wind speed was varied from 0 to $16 \mathrm{~m} / \mathrm{s}$, and 18 different wind speeds were used.

\footnotetext{
${ }^{a}$ Corresponding author: kimyc@arch.t-kougei.ac.jp

Part of this paper was presented at The $15^{\text {th }}$ International Conference Civil, Structural and Environmental Computing in Prague, Czech.
}

\section{Outlines of wind tunnel experiment}

The solar wing model is shown in Figure 1. It comprises 12 solar wing units spaced at equal intervals by two cables. Each solar wing unit comprises 6 solar panel modules, but in the experiment they were simply represented by one wooden C-shaped plate. The length scale was $1 / 33.33$, and the velocity scale was $1 / 3.65$. The mass scale, which is the third power of the length scale, is $1 / 2369$, and the mass of one wooden plate was determined considering the mass of one solar wing unit, which included the mass of 6 solar panel modules and module supporters, and 2 cables. The cross section of the cables was determined considering elastic parameters, and in the experiment piano wires with diameter of $0.56 \mathrm{~mm}$ were used.

Three laser displacement transducers were installed under a turntable to measure the vertical displacements $y$ of solar wing units 3, 6, and 10, as shown in Figure 1 (simply, P3, P6, and P10). The sampling frequency of the laser displacement transducers was $300 \mathrm{~Hz}$, and the measuring time was adjusted such that one 10-minute sample was obtained. Wind speeds increased from $0 \mathrm{~m} / \mathrm{s}$ to $16 \mathrm{~m} / \mathrm{s}$ with 18 intervals. In this wind speed range, the Froude number and elastic parameter can cover those of full scale. Strain gauges were also installed in column A and column B. The sag ratio was defined as the ratio of sag at the middle of the cable to the span length, and two sag ratios, $2 \%$ and $5 \%$, were considered in the experiment. When the sag ratio was $2 \%$, a triangular fairing was added to the most windward side solar wing unit. Basically, wind directions $\theta$ from $0^{\circ}$ to $90^{\circ}$ were 
employed. The natural frequency of the vertical direction was about $4.1 \mathrm{~Hz}$, and the system was designed to have a very low damping ratio as soon as possible.

The wind tunnel experiment was conducted under a uniform flow, and to eliminate the effects of the supporting structures on the turntable, tapers were installed at four sides of the supporting structures, and the inside of the supporting structures was also covered by a light board.

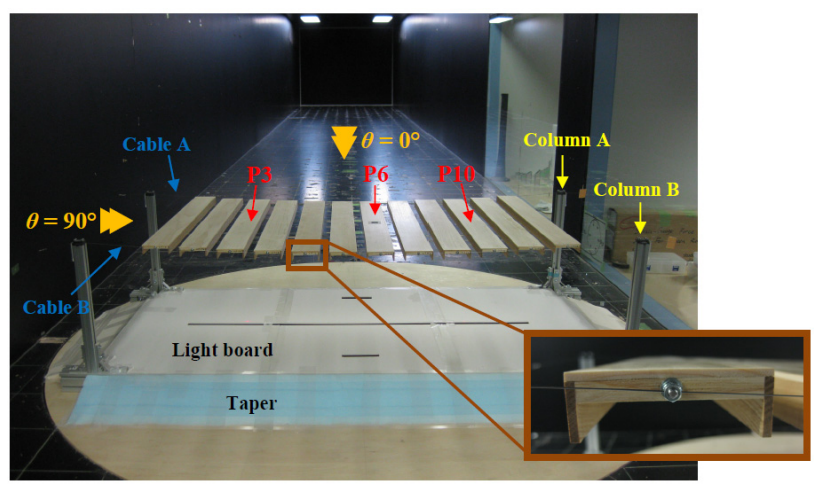

Figure 1. Setting of solar wind model $\left(\theta=0^{\circ}\right)$.

\section{Results and discussions}

\subsection{Vertical displacement}

Figure 2 shows the definitions of vertical displacements $y$ for each solar unit. Downward displacement was defined as positive, and mean $y_{\text {mean }}$ and standard deviation $y_{\text {std }}$ of each solar unit were obtained from a time series of vertical displacements.

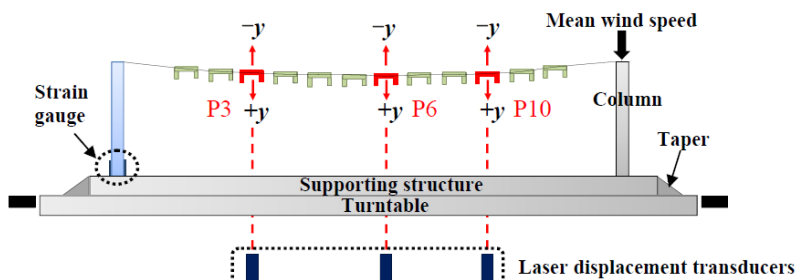

Figure 2. Definition of vertical displacement $y$ (Downward is positive, $\theta=0^{\circ}$ ).

In Figure 3, variations of mean and fluctuating vertical displacements with mean wind speed for wind directions $\theta=0^{\circ}$ and $30^{\circ}$ are shown when sag is $2 \%$. Mean wind speeds were measured at the column tips as shown in Figure 2, and displacement and mean wind speed are model scale values. Absolute values of mean vertical displacements $y_{\text {mean }}$ (Figure 3(a)) increase with mean wind speeds and increase until wind direction $\theta=$ $30^{\circ}$. Negative displacements indicate that the solar units shifted to the upward direction. In this study, increase (or large) and decrease (or small) mean that the absolute value increase (is large) and decrease (is small) regardless of their sign for convenience. Differences among solar units seem to be small even though slightly larger values are shown for P6 at wind direction $\theta=30^{\circ}$. Fluctuating vertical displacements shown in Figure 3(b) increase monotonically with mean wind speed and also increase with wind direction.

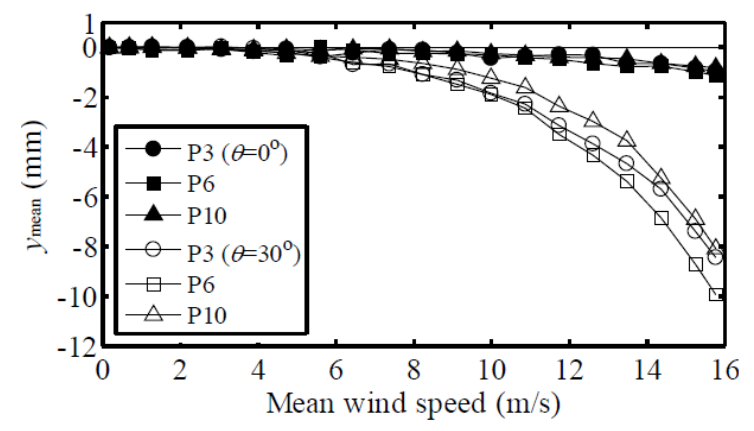

(a) Mean vertical displacement $y_{\text {mean }}$

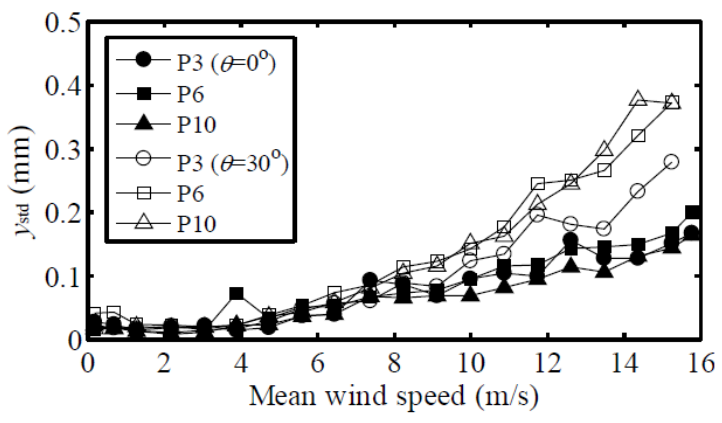

(b) Fluctuating vertical displacement $y_{\text {std }}$

Figure 3. Variations of vertical displacements at $\theta=0^{\circ}$ and $30^{\circ}$ when sag is $2 \%$.

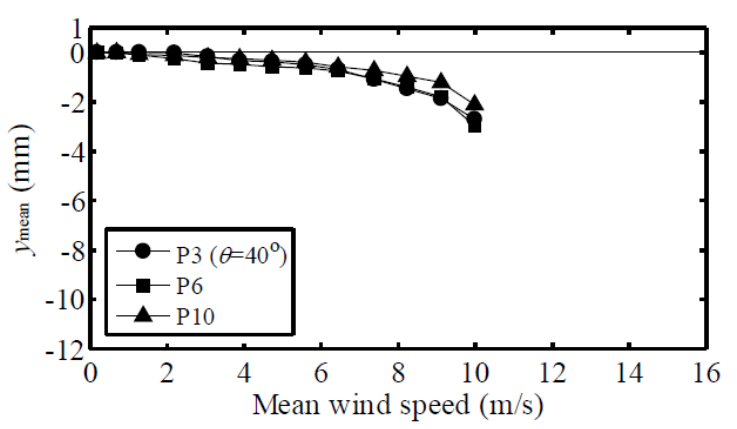

(a) Mean vertical displacement $y_{\text {mean }}$

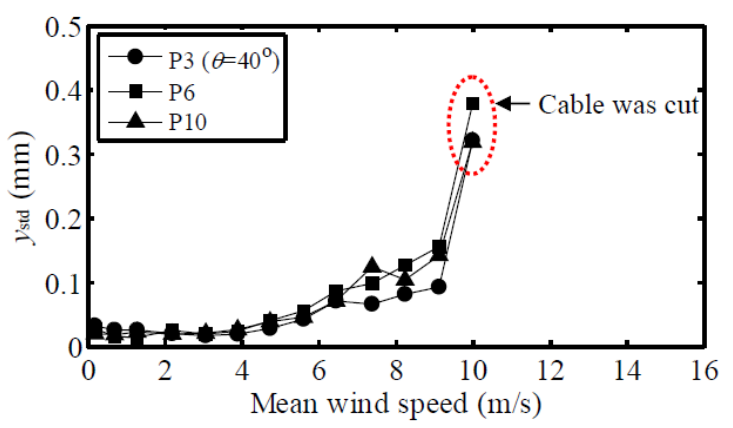

(b) Fluctuating vertical displacement $y_{\text {std }}$

Figure 4. Variations of vertical displacements at $\theta=40^{\circ}$ and $30^{\circ}$ when sag is $2 \%$.

Vertical displacements at wind direction $\theta=40^{\circ}$ are shown in Figure 4. The mean vertical displacements shown in Figure 4(a) increase gradually with mean wind speed, showing a similar trend and a little larger values to those of $\theta=30^{\circ}$. But the fluctuating displacements (Figure 4(b)) increase suddenly near the mean wind speed 
of $10 \mathrm{~m} / \mathrm{s}$, and at this mean wind speed one of the cables was cut because of severe vibrations. Thus, for the following experiments, when severe vibrations were observed in any of solar units, the experiment was stopped.

Figure 5 shows the vertical displacements for wind direction $\theta=90^{\circ}$. The mean vertical displacements increase monotonically (Figure 5(a)), but for the fluctuating vertical displacements (Figure 5(b)), a large and sudden increase is observed near the mean wind speed of $1 \mathrm{~m} / \mathrm{s}$. But when the mean wind speed increases a little, i.e., $2 \mathrm{~m} / \mathrm{s}$, this large and sudden increase is not observed and they increase monotonically with mean wind speed. This phenomenon is clearly shown when the wind direction is larger than $60^{\circ}$. The mean displacements decrease when the solar unit is located on the downstream side (smallest for P10), but the fluctuating displacements increase when the solar unit is located on the downstream side (largest for the P10). Near the mean wind speed of $6 \mathrm{~m} / \mathrm{s}$, as the vertical displacement of P10, which is located on the downstream side, increases largely, and the experiment was stopped.

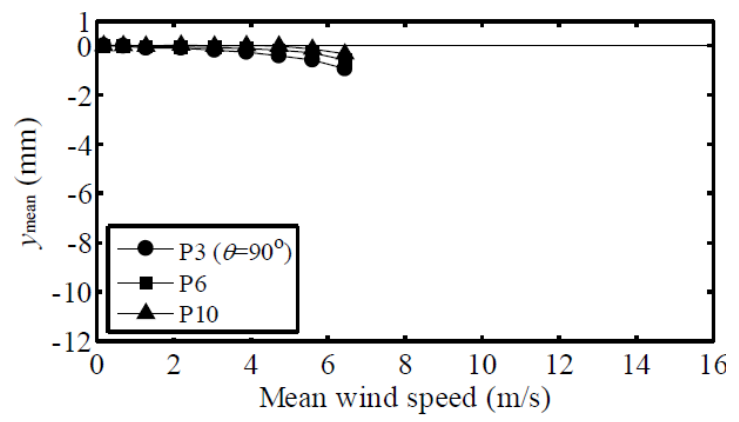

(a) Mean vertical displacement $y_{\text {mean }}$

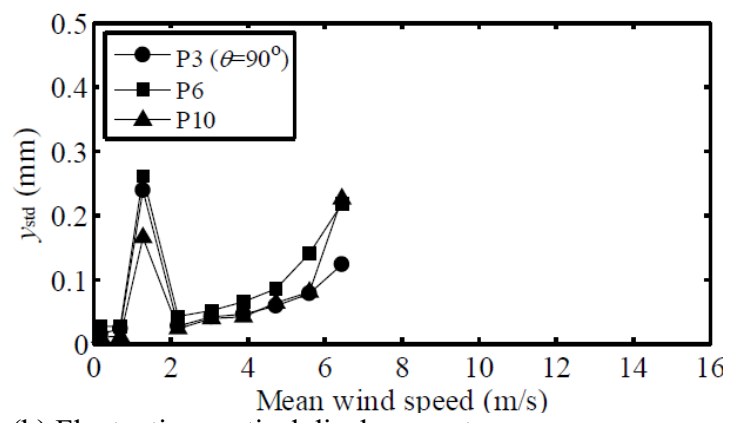

(b) Fluctuating vertical displacement $y_{\text {std }}$

Figure 5. Variations of vertical displacements at $\theta=90^{\circ}$ and $30^{\circ}$ when sag is $2 \%$.

When the sag is $2 \%$, both mean and fluctuating displacements increase gradually up to wind direction $\theta=$ $50^{\circ}$. The mean displacements shows similar values and trends after that, but clear differences were observed in fluctuating displacements, resulting from the characteristic vibrations with wind direction. Due to the characteristic vibrations, the experiments were stopped at a smaller mean wind speed for large wind directions. Differences among solar units were not clearly identified, and all solar units shifted in the upward direction.

In Figure 6, the variations of vertical displacements with mean wind speed at wind directions of $\theta=0^{\circ}$ and $20^{\circ}$ are shown when sag is $5 \%$. At wind direction $\theta=0^{\circ}$, the mean vertical displacements (Figure 6(a)) are larger than those when sag is $2 \%$, and at wind direction $\theta=20^{\circ}$, large differences are found among solar units, showing the largest value for P3. Note that the vertical displacements of $\mathrm{P} 10$ at wind direction $\theta=20^{\circ}$ show positive values, implying that they shifted to the downward direction. This trend is found for all wind directions except $\theta=0^{\circ}$. The fluctuating displacements (Figure 6(b)) show a linearly increasing trend with mean wind speed, showing smaller values than those when the sag is $2 \%$. Differences among solar units are not clear for these wind directions.

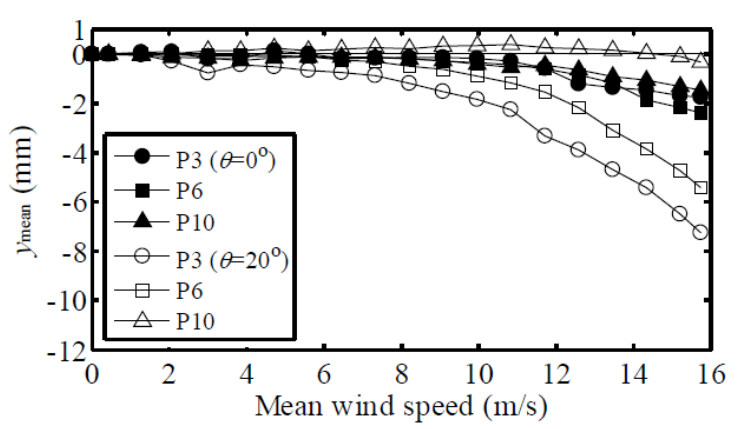

(a) Mean vertical displacement $y_{\text {mean }}$

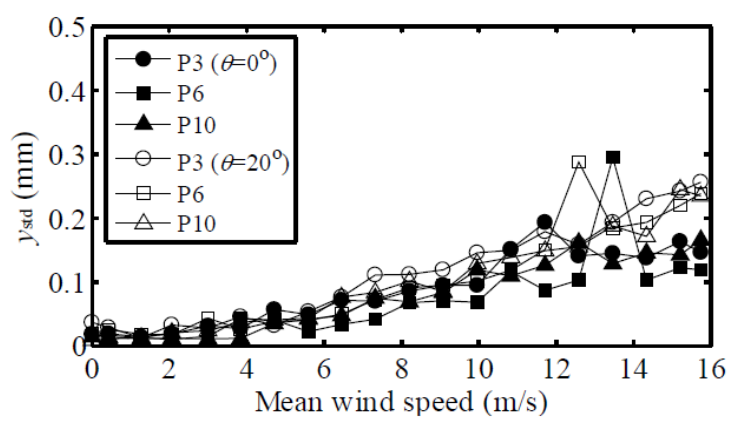

(b) Fluctuating vertical displacement $y_{\text {std }}$

Figure 6. Variations of vertical displacements at $\theta=0^{\circ}$ and $20^{\circ}$ when sag is $5 \%$.

Vertical displacements at wind direction $\theta=90^{\circ}$ are shown in Figure 7. The mean displacements (Figure 7(a)) of P10 show positive values as mentioned before, and quite complicated variations are found in the fluctuating displacements as shown in Figure 7(b). Unlike for the sag of $2 \%$, sudden increase near the mean wind speed of $1 \mathrm{~m} / \mathrm{s}$ was not clearly identified and rather complicate variations were observed.

As shown in Figure 5, a sudden increase in fluctuating displacements is found near the mean wind speed of $1 \mathrm{~m} / \mathrm{s}$ when the sag is $2 \%$ and to reduce this vibration, a fairing was tentatively added to the most upwind solar unit. The shape and dimensions of the fairing are shown in Figure $8(\mathrm{a})$, and the results are shown in Figure 8(b). With current shape and dimensions, no suppressions were expected as shown in Figure 8(b), and conversely the fluctuating displacements of P6 (Center solar unit) are slightly larger than those without the fairing. 


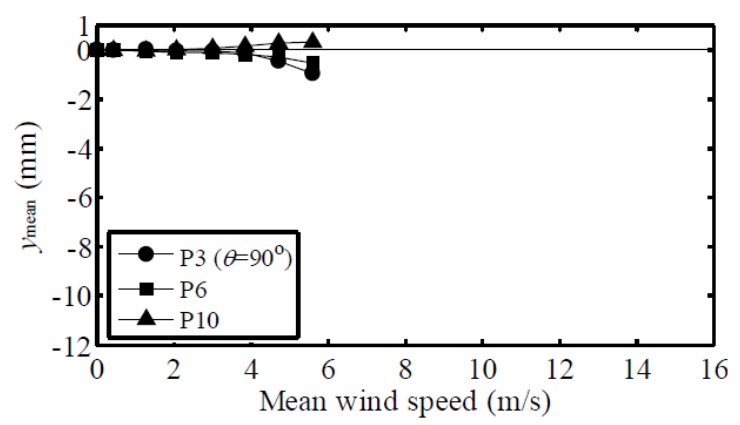

(a) Mean vertical displacement $y_{\text {mean }}$

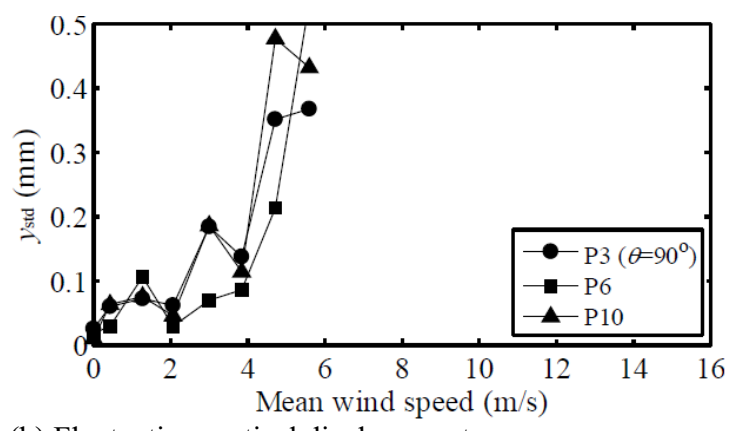

(b) Fluctuating vertical displacement $y_{\text {std }}$

Figure 7. Variations of vertical displacements at $\theta=90^{\circ}$ when sag is $5 \%$.

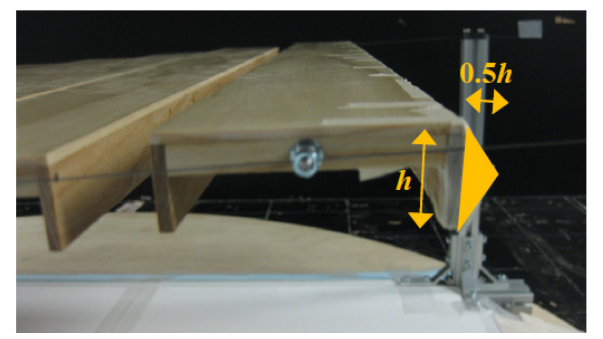

(a) Shape and dimension of fairing.

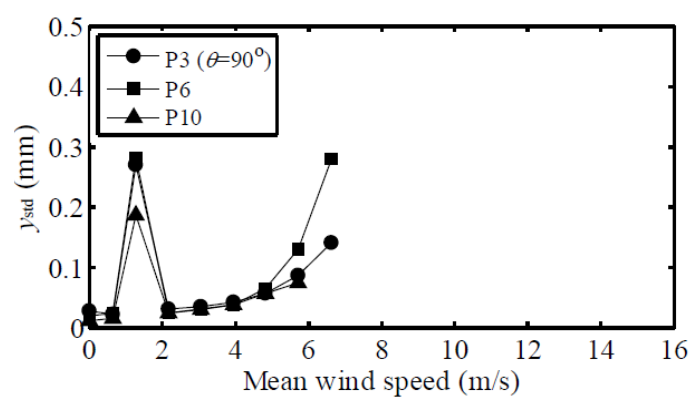

(b) Fluctuating vertical displacement $y_{\text {std }}$

Figure 8. Variations of vertical displacements at $\theta=90^{\circ}$ when sag is $5 \%$.

Additional experiment was conducted to examine the cause of the sudden increase in fluctuating displacement at the wind direction of $\theta=90^{\circ}$. The experimental method is as follows. As shown in Figure 9, firstly, all intervals between solar units were covered by light boards, and vertical displacements were measured. When all intervals between solar wing units were covered, no sudden increase was observed, meaning that the intervals between solar units play an important role in such an unstable vibration. And then, just light board 1) was removed, remaining the rest of light board, and the displacements were measured. After that again just light board 2) was removed, remaining the rest of light board, and measure the displacements. Like this way, the lights boards were removed one by one, and the results were shown in Table 1.

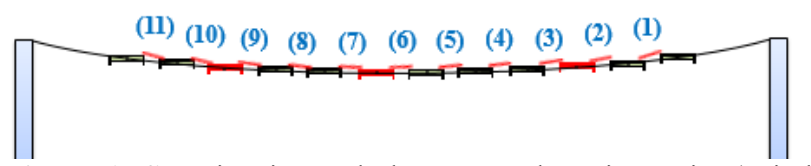

Figure 9. Covering intervals between solar wing units (Wind blows from left to right).

Table 1. Position of light board and observation of unstable vibration.

\begin{tabular}{|c|c|}
\hline Position of light board & Observation of unstable vibration \\
\hline 1$) \sim 11)$ & No \\
\hline 2$) \sim 11)$ & No \\
\hline 3$) \sim 11)$ & No \\
\hline 4$) \sim 11)$ & No \\
\hline 5$) \sim 11)$ & No \\
\hline 6$) \sim 11)$ & No \\
\hline 7$) \sim 11)$ & No \\
\hline 8$) \sim 11)$ & No \\
\hline 9$) \sim 11)$ & No \\
\hline 10$) \sim 11)$ & No \\
\hline 11$)$ & Yes \\
\hline 10$)$ & Yes \\
\hline No light boards & Yes \\
\hline
\end{tabular}

As shown in Table 1, it was found that when one of the intervals of 10) and 11) or both are not covered regardless of other intervals, the sudden increase in fluctuating vibrations was observed. From the visualization, the shear layer separated from the P10 and/or P11 flowed out under the P11 and/or P12, after impinging the windward surface of P11 and P12. Although it was not clearly identified, vortex seems to form inside the intervals between P10 and P11 (Interval 10)), and P11 and P12 (Interval 11)), implying that the unstable vibration is caused by the vortex formed inside the intervals. This kind of unstable vibration is known as edge tone, which is characterized by another type of flow instability caused by the vortex shedding from the elongated bluff body. Unlike the Karman vortex, where the two separated shear layers interact with each other behind the bluff body to from a staggered vortex streets, edge tone or impinging-shear-layer instability resulted from a single separated shear layer from the elongated bluff body by the presence of an impingement edge in the downstream side $[3,4,5]$. The possible mechanisms are shown in Figure 10. Figure 10(a) shows the first possible mechanism that separated shear layer issuing from the upwind panel (P10 or P11) intersects with the sharp corner of the downwind panel (P11 or P12), occuring the instability vibration. In this case, the representative width is $d_{l}$ as shown in Figure 10(a). The second possible mechanism is shown in Figure 10(b). The flows flowing into the interval 10 or interval 11 separate at the leading 
edge corner of the $\mathrm{P} 11$ or $\mathrm{P} 12$, and intersect with the trailig edge corner of the same panels. For the second mechanism, the inner distance of vertical plate $d$ is the representative width.

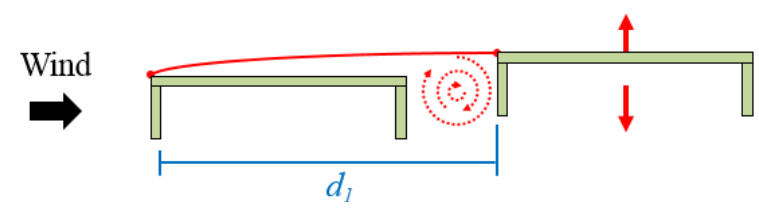

(a) Mechanism 1

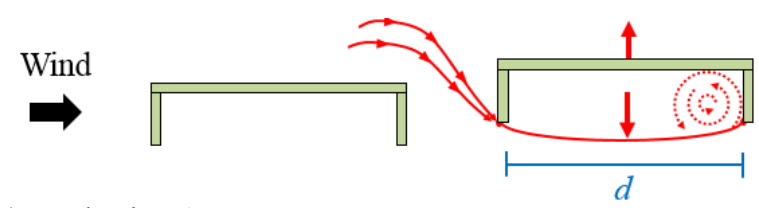

(b) Mechanism 2

Figure 10. Possible mechanisms of edge tone.

\subsection{Tension of cables}

Strain gauges were installed in columns A and B (Figure $1)$, and the measured strains were transformed to the tension $T$ in cable A and cable B (Figure 1). When the tension $T$ is applied in the inward direction, the cables are displaced in the downward direction, which is defined as positive tension. Conversely, negative tension is defined when the tension $T$ is applied in the outward direction, displacing the cables in the upward direction.

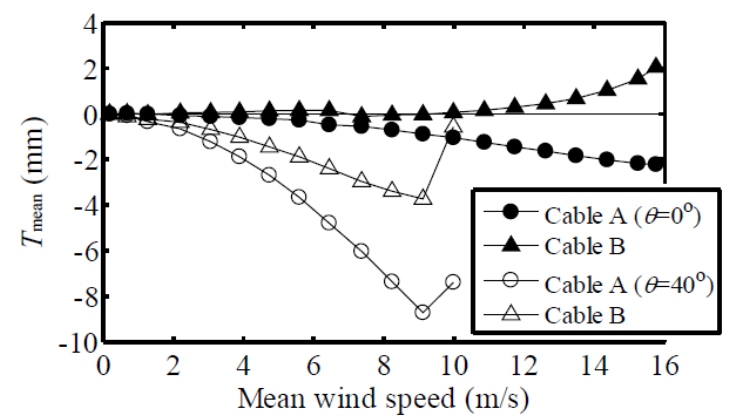

(a) Mean tension $T_{\text {mean }}$

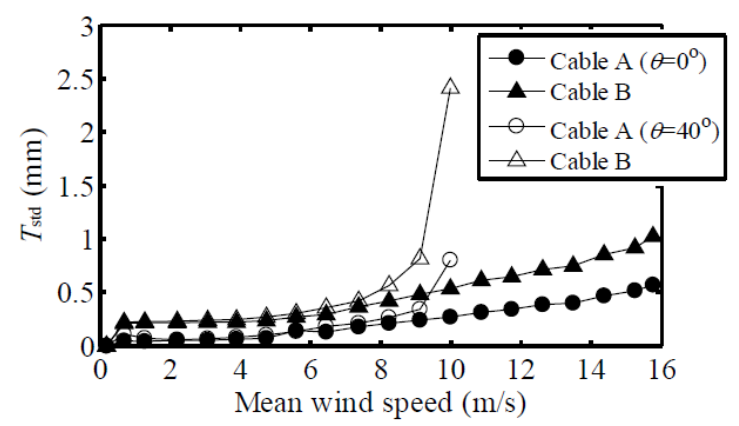

(b) Fluctuating tension $T_{\text {std }}$

Figure 11. Variation of tension at $\theta=0^{\circ}$ and $\theta=40^{\circ}$ when sag is $2 \%$.

The variations of mean and fluctuating tension at wind directions $\theta=0^{\circ}$ and $\theta=40^{\circ}$ when sag is $2 \%$ are shown in Figure 11. When wind direction $\theta=0^{\circ}$, cable A, which is located on the windward side, shows negative tensions and cable $\mathrm{B}$, which is located on the leeward side, shows positive tensions (Figure 11(a)). This means that the solar units are rotated counter-clockwise, and are not in the initial parallel condition. As wind direction increases, mean tensions in cable B become negative and mean tensions in both cables increase up to wind direction $\theta=40^{\circ}$ or $50^{\circ}$. At wind direction $\theta=40^{\circ}$ a sudden decrease in mean tensions and a sudden increase in fluctuating tensions were found near the mean wind speed of $10 \mathrm{~m} / \mathrm{s}$ (Figure 11(b)), which corresponds to the sudden increase in fluctuating vertical displacement as shown in Figure 4.

At wind direction $\theta=90^{\circ}$, the mean tensions in both cables vary little with mean wind speed, staying in the initial parallel condition (Figure 12).

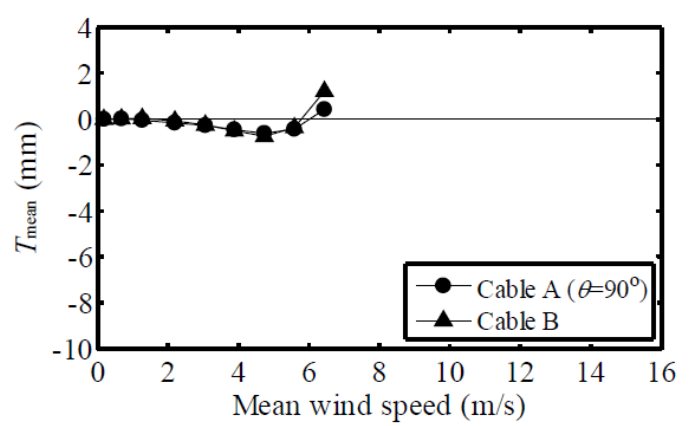

Figure 12. Variation of mean tension $T_{\text {mean }}$ at $\theta=90^{\circ}$ when sag is $2 \%$.

\section{Concluding remarks}

Through wind tunnel experiments, the following concluding remarks can be made.

When the sag is $2 \%$, the vertical displacements increase monotonically with mean wind speed, showing no characteristic vibrations when the wind directions are between $0^{\circ}$ and $30^{\circ}$. A sudden increase in fluctuating displacement was found near mean wind speed $10 \mathrm{~m} / \mathrm{s}$ at wind direction $40^{\circ}$, cutting the cable. This means that, for a certain wind direction, a solar wing system may collapse suddenly because of large vibration. A sudden increase in fluctuating displacements was also found near mean wind speed $1 \mathrm{~m} / \mathrm{s}$ when the wind direction was larger than $60^{\circ}$.

When the sag increases to $5 \%$, clear differences among solar wing units in mean displacements were found and complicate vibration in fluctuating displacement at low wind speed was observed. When the sag was $2 \%$, solar wing units shifted to the upwind direction, showing negative displacements, but when the sag was 5\%, mean displacements of P10 becomes positive, meaning that it shifted to the downward direction for wide ranges of wind directions.

\section{References}

1. F.P. Baumgartner, A. Büchel, R. Bartholet, Proceedings of the 24th European Photovoltaic Solar Energy Conference (Hamburg, 2009).

2. F.P. Baumgartner, A. Büchel, R. Bartholet, Proceedings of the 25th European Photovoltaic Solar Energy Conference (Valencia, 2010). 
3. N. Nakayama, Y. Ohya, S. Ozono, R. Nakayama, J. Wind Eng. Ind. Aerodynamics 65, 301-308 (1996).

4. E. Naudascher, D. Rockwell, Flow-induced vibration, An engineering guide (Dover Publications, New York, 2005).

5. Y. Nakamura, M. Nakashima, J. Fluid Mech. 163, 149-169 (1986). 